\title{
Development and evaluation of an up- converting phosphor technology-based lateral flow assay for rapid and quantitative detection of Coxiella burnetii phase I strains
}

Pingping Zhang ${ }^{1,2+}$, Jun Jiao ${ }^{1,2+}$, Yong Zhao ${ }^{1,2}$, Mengjiao Fu ${ }^{1,2}$, Jin Wang ${ }^{1,2}$, Yajun Song ${ }^{1,2}$, Dongsheng Zhou ${ }^{1,2}$, Yongqiang Wang ${ }^{3}$, Bohai Wen ${ }^{1,2}$, Ruifu Yang ${ }^{1,2^{*}}$ and Xiaolu Xiong ${ }^{1,2^{*}}$

\begin{abstract}
Background: Coxiella burnetii is an obligate intracellular Gram-negative bacterium that causes a zoonotic disease commonly called Q fever globally. In this study, an up-converting phosphor technology-based lateral flow (UPT-LF) assay was established for the rapid and specific detection of phase I strains of $C$. burnetii.

Results: Specific monoclonal antibodies (10B5 and 10G7) against C. burnetii phase I strains were prepared and selected for use in the UPT-LF assay by the double-antibody-sandwich method. The detection sensitivity of the Coxiella-UPT-LF was $5 \times 10^{4} \mathrm{GE} / \mathrm{ml}$ for a purified C. burnetii phase I strain and $10 \mathrm{ng} / \mathrm{ml}$ for LPS of C. burnetii Nine Mile phase I (NMI). Good linearity was observed for C. burnetii phase I and NMI LPS quantification $\left(R^{2} \geq 0.989\right)$. The UPT-LF assay also exhibited a high specificity to C. burnetii, without false-positive results even at $10^{8} \mathrm{GE} / \mathrm{ml}$ of non-specific bacteria, and good inclusivity for detecting different phase I strains of C. burnetii. Moreover, the performance of the Coxiella-UPT-LF assay was further confirmed using experimentally and naturally infected samples.
\end{abstract}

Conclusions: Our results indicate that Coxiella-UPT-LF is a sensitive and reliable method for rapid screening of $C$. burnetii, suitable for on-site detection in the field.

Keywords: Coxiella burnetii, Q fever, Lipopolysaccharide, Up-converting phosphor technology-based lateral flow, Monoclonal antibody

\section{Background}

Coxiella burnetii is an intracellular Gram-negative bacterium that causes a zoonotic disease known as Q fever globally. It can undergo a phase transition that is correlated with some of the biological characteristics of the "smooth-to-rough" lipopolysaccharide (LPS) variation observed for Gram-negative Enterobacteriaceae [1]. The virulent form of C. burnetii (phase I, PI) has full-length

\footnotetext{
*Correspondence: ruifuyang@gmail.com; xiongxiaolu624@sohu.com

${ }^{\dagger}$ Pingping Zhang and Jun Jiao contributed equally to this work.

${ }^{1}$ State Key Laboratory of Pathogen and Biosecurity, Beijing Institute of Microbiology and Epidemiology, Beijing, P. R. China

Full list of author information is available at the end of the article
}

LPS and is usually isolated from natural and laboratory infections [2]. Upon serially passaging in embryonic cells, tissue culture, or synthetic medium, a smooth-torough (truncated) LPS transition occurs, which results in avirulence (phase II, PII) [3]. C. burnetii PI is able to replicate in immunocompetent hosts and is considered as a category B bio-warfare agent [4], which makes it a public health and biosecurity concern.

Diagnosis of $\mathrm{Q}$ fever is difficult due to the lack of distinct clinical features that distinguish it from other febrile diseases [5]. Currently, the diagnosis of Q fever mainly depends on the detection of antibodies or nucleic

(c) The Author(s). 2020 Open Access This article is licensed under a Creative Commons Attribution 4.0 International License, which permits use, sharing, adaptation, distribution and reproduction in any medium or format, as long as you give appropriate credit to the original author(s) and the source, provide a link to the Creative Commons licence, and indicate if changes were made. The images or other third party material in this article are included in the article's Creative Commons licence, unless indicated otherwise in a credit line to the material. If material is not included in the article's Creative Commons licence and your intended use is not permitted by statutory regulation or exceeds the permitted use, you will need to obtain permission directly from the copyright holder. To view a copy of this licence, visit http://creativecommons.org/licenses/by/4.0/ The Creative Commons Public Domain Dedication waiver (http://creativecommons.org/publicdomain/zero/1.0/) applies to the data made available in this article, unless otherwise stated in a credit line to the data. 
acids. Serological methods, especially immunofluorescence assays, are considered as the reference methods for diagnosis of $\mathrm{Q}$ fever in both humans and animals. However, an important drawback to serological diagnosis of acute $\mathrm{Q}$ fever is the lag phase in antibody response of 7-15 days after onset of clinical symptoms, limiting early diagnosis [6]. Although PCR-based approaches provide a more sensitive way for the detection of $\mathrm{Q}$ fever, the results of PCR testing of peripheral blood are variable and do not provide information on the viability of $C$. burnetii $[7,8]$. Moreover, the need for expensive equipment and professional training is also a barrier for the use of these methods in primary laboratories and in the field.

To prevent or minimise Q fever outbreaks in humans, rapid, simple, sensitive, and accurate methods for $C$. burnetii detection in natural infections and for potential bioterrorist attacks are still needed. Recently, an upconverting phosphor technology-based lateral flow (UPT-LF) assay using up-converting phosphor particles (UCPs) as the bio-label, with excitation and emission peaks at 980 and $541.5 \mathrm{~nm}$, has been developed as a new point-of-care testing method. UPT-LF exhibits high sensitivity and stability, as well as robust performance when tested with complex samples [9-12]. In the current study, a UPT-LF assay for the rapid and specific detection of PI strains of C. burnetii was established. The performance of this assay was comprehensively evaluated with cultured material and experimentally and naturally infected samples.

\section{Results}

\section{Development of Coxiella-UPT-LF}

The monoclonal antibodies (mAbs) against C. burnetii were prepared in mice that were immunised with purified C. burnetii Xinqiao strain (PI). Three cloned hybridomas (10B5, 10G7, and 13D6) that produced C. burnetii PI-specific $\mathrm{mAb}$ and two cloned hybridomas (6D8 and 8A1) that produced both PI- and PII-specific mAb were identified by ELISA analysis of hybridoma supernatants with PI and PII antigens. The isotypes, concentrations, and potencies of these mAbs are listed in Table 1.
To screen for suitable antibodies for the UPT-LF assay, strips fabricated with conjugation pads and nitrocellulose membranes with various antibodies were evaluated using a C. burnetii Xinqiao strain purified from yolk sac (YS) with primary sample treating buffer and different labelling conditions between UCPs and antibodies. The results are shown in Fig. 1. Strips with nitrocellulose membrane with 10B5, 10G7, and 13D6 paired with conjugation pads with 10G7 or 10B5 showed excellent performances. After further optimisation of the sample treating buffer components, as well as optimisation of labelling conditions between UCP and antibodies, Coxiella-UPT-LF strips with $10 \mathrm{G} 7$ on the nitrocellulose membrane and 10B5 on the conjugation pad were finally selected; the corresponding sample treating buffer was $0.03 \mathrm{M}$ phosphate buffer containing $0.5 \%$ IGEPAL CA-630 and $0.1 \mathrm{M} \mathrm{NaCl}$.

The amino acid sequences of variable heavy $(\mathrm{VH})$ and variable light $(\mathrm{VL})$ chains of 10B5 and 10G7 were determined by cDNA sequencing. The sequences of the $\mathrm{VH}$ and VL chains of 10B5 and 10G7 were identical, indicating that they were subclones from the same fusion event (Fig. S1).

\section{Detection limit, sensitivity, and precision}

Samples with purified C. burnetii Xinqiao strain at concentrations from $1 \times 10^{3}$ to $1 \times 10^{8}$ genome equivalents (GE)/ml or Nine Mile PI (NMI) or PII (NMII) LPS from 1 to $10,000 \mathrm{ng} / \mathrm{ml}$ were analysed in triplicate. Phosphate buffer was measured 10 times, and the mean plus three standard deviations was set as the cut-off value, which was 0.097. Samples with a T/C ratio higher than the cut-off value were considered as positive. The lowest concentration with at least one positive result higher than the cutoff was defined as the detection limit of Coxiella-UPT-LF; the lowest concentration with three positive results out of three tests was defined as the sensitivity.

The detection limit and sensitivity of Coxiella-UPT-LF for purified C. burnetii Xinqiao strain are $1 \times 10^{4}$ and $5 \times 10^{4} \mathrm{GE} / \mathrm{ml}$, respectively. The detection limit and sensitivity of NMI LPS are 1 and $10 \mathrm{ng} / \mathrm{ml}$, respectively. Coefficients of variation of Coxiella-UPT-LF for each concentration are defined as the ratios of standard

Table 1 The monoclonal antibodies against C. burnetii

\begin{tabular}{|c|c|c|c|c|}
\hline \multirow[t]{2}{*}{ Antibody } & \multirow[t]{2}{*}{ Isotype } & \multicolumn{2}{|l|}{ Potency } & \multirow{2}{*}{$\begin{array}{l}\text { Concentration } \\
(\mathrm{mg} / \mathrm{ml})\end{array}$} \\
\hline & & $\begin{array}{l}\text { Pl-specific mAb } \\
\text { (PI Xinqiao strain) }\end{array}$ & PII-specific mAb (PII Grita strain) & \\
\hline 10B5 & $\lg M$ & $62.5 \mathrm{ng} / \mathrm{ml}$ & - & 8 \\
\hline 10G7 & $\lg M$ & $31.2 \mathrm{ng} / \mathrm{ml}$ & - & 5 \\
\hline 13D6 & $\lg G 1$ & $15.6 \mathrm{ng} / \mathrm{ml}$ & - & 10 \\
\hline 6D8 & $\lg M$ & $125 \mathrm{ng} / \mathrm{ml}$ & $125 \mathrm{ng} / \mathrm{ml}$ & 6 \\
\hline $8 \mathrm{~A} 1$ & $\lg G 2 b$ & $62.5 \mathrm{ng} / \mathrm{ml}$ & $7.8 \mathrm{ng} / \mathrm{ml}$ & 14 \\
\hline
\end{tabular}




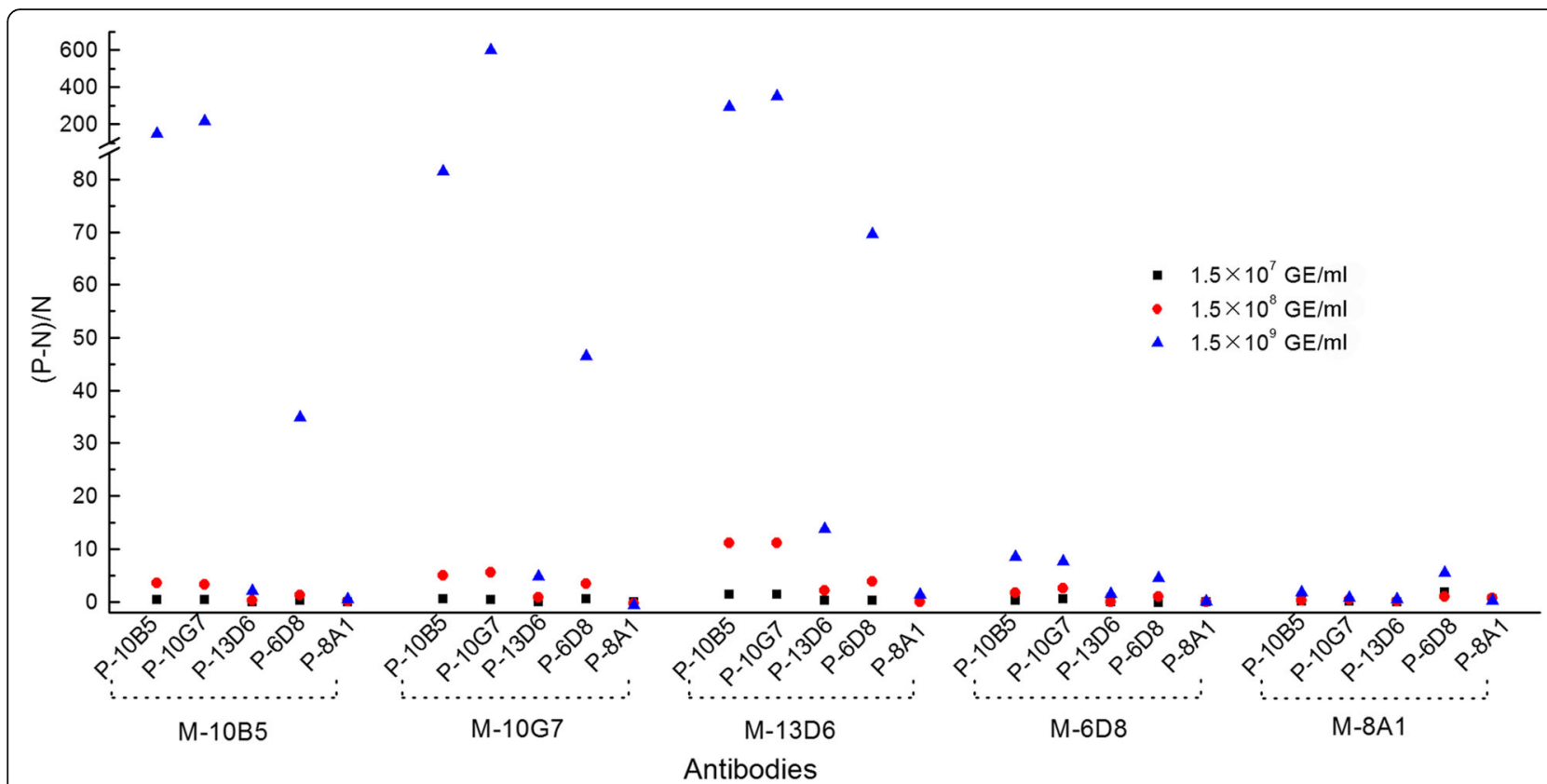

Fig. 1 Assessment of UPT-LF strips fabricated using different antibodies. Nitrocellulose membranes and conjugation pads both with various antibodies were paired randomly in the fabrications of the strips, and each strip was used for the detection of $C$. burnetii Xinqiao strain at three different concentrations with primary sample treating buffer and different labelling conditions between UCPs and antibodies. The letter "P" before the name of the antibodies means that the antibodies were on the conjugate pad, while the letter " $\mathrm{M}$ " means they were on the nitrocellulose membrane

deviations and means; they were all less than $15 \%$ for the detection of purified C. burnetii Xinqiao strain with concentrations below $5 \times 10^{7} \mathrm{GE} / \mathrm{ml}$ and the detection of NMI LPS with concentrations below $1000 \mathrm{ng} / \mathrm{ml}$. With increasing concentrations, the signals on the $\mathrm{C}$ band were too high for exact detection, which further influenced the precision of the strip. As expected, Coxiella-UPT-LF did not react with NMII or NMII LPS at any of the concentrations mentioned above. The specificity of mAbs to PI LPS was further confirmed by immunoblot with LPS extracted from different C. burnetii strains. As shown in Fig. 2d (also shown in Fig. S2 and Fig. S3), mAbs pooled from 10B5 and 10G7 reacted with NMI LPS, as indicated by a laddering profile above $14 \mathrm{kDa}$, but did not react with NMII LPS.

For linear quantitative correlation analysis, a standard curve was plotted for Coxiella-UPT-LF for purified $C$. burnetii Xinqiao strain from $1 \times 10^{4}$ to $1 \times 10^{8} \mathrm{GE} / \mathrm{ml}$, as well as for NMI LPS from 10 to $10,000 \mathrm{ng} / \mathrm{ml}$ (Fig. 2e and $\mathrm{f}$ ), with the logarithm of the difference between the $\mathrm{T} / \mathrm{C}$ ratio and the cut-off value on the horizontal axis and the logarithm of the concentration on the vertical axis. The $\mathrm{R}$ values of the curves for purified $C$. burnetii Xinqiao and NMI LPS were both larger than 0.9, demonstrating the excellent accuracy for quantification.

\section{Specificity}

Various purified bacteria with a concentration of $10^{8}$ $\mathrm{GE} / \mathrm{ml}$ were used to evaluate the specificity of Coxiella-
UPT-LF, including 8 genetically related bacteria, 4 bioterrorism agents, and 15 food-borne bacteria that have similar habitats as C. burnetii. As shown in Fig. 3, Coxiella-UPT-LF only exhibited slight cross-reaction with Vibrio cholerae $\mathrm{O} 139$ at a concentration of $10^{8} \mathrm{GE} /$ $\mathrm{ml}$. When $V$. cholerae $\mathrm{O} 139$ was added to the strip at a concentration of $10^{7} \mathrm{GE} / \mathrm{ml}$, no cross-reaction was observed, indicating that Coxiella-UPT-LF has high specificity to C. burnetii.

\section{Inclusivity}

Five C. burnetii strains in YS suspension were used to assess the inclusivity of Coxiella-UPT-LF, i.e., three PI strains (C. burnetii Qiyi, Yaan, and $\mathrm{H}-11$ ) and two PII strains (C. burnetii Grita and NMII). C. burnetii Xinqiao strain purified from YS served as a positive control. As negative controls, 10-, 100-, 1000-, and 10,000-fold dilutions of YS suspension and phosphate buffer were used. All of the negative controls had little influence on the detection of Coxiella-UPT-LF. As shown in Fig. 4a, the sensitivity of Coxiella-UPT-LF for C. burnetii Qiyi, Yaan, and $\mathrm{H}-11$ in YS suspension was less than $1 \times 10^{6}, 1 \times$ $10^{6}$, and $1 \times 10^{7} \mathrm{GE} / \mathrm{ml}$, respectively, and Coxiella-UPTLF did not react with C. burnetii NMII and Grita, the strains of avirulent forms. The inclusivity of CoxiellaUPT-LF for PI C. burnetii and its non-reactivity with avirulent forms has practical significance for C. burnetii detection in the field. 
a

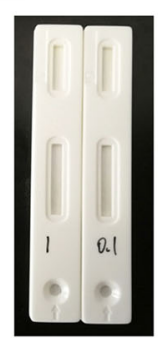

C

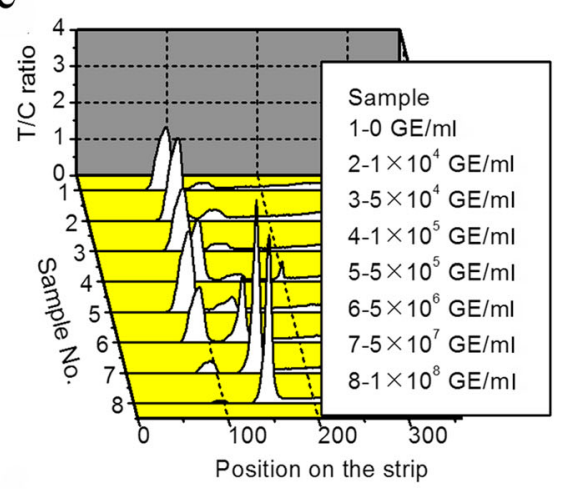

$\mathrm{e}$

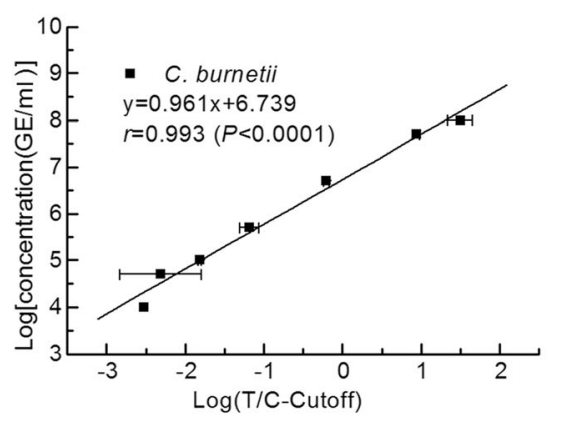

b

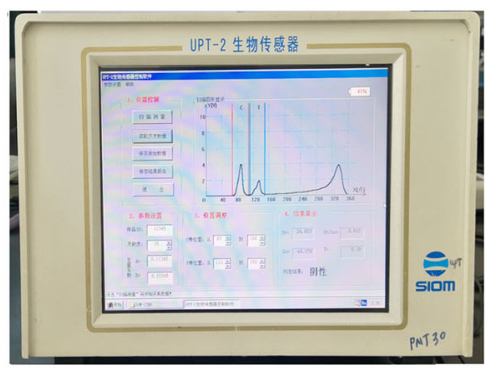

d

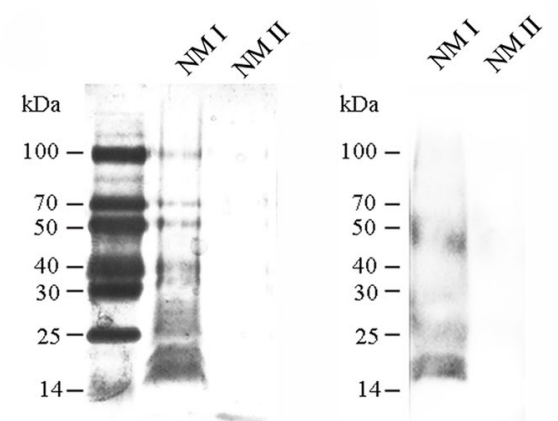

f

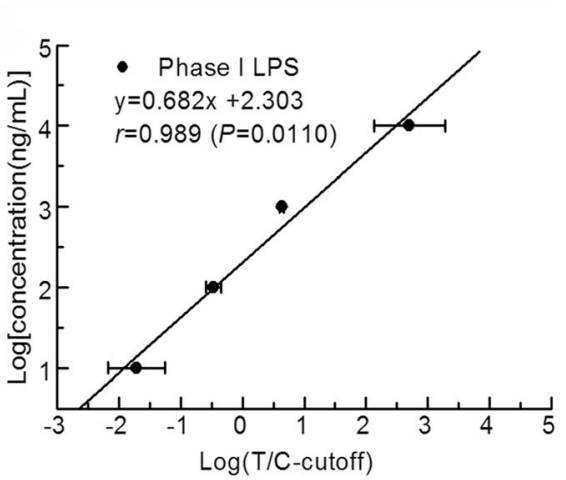

Fig. 2 The detection limit, sensitivity, and precision of Coxiella-UPT-LF. a Photograph of a UPT-LF strip. b Photograph of the UPT biosensor. c Illustrations of the UPT assay for the detection of C. burnetii Xinqiao strain. Peaks on the left are signals for the control bands, and peaks on the right are for test bands. $\mathbf{d}$ LPS immunoblot of C. burnetii NMI and NMII. LPS of C. burnetii NMI and NMII was separated by SDS-PAGE, silver-stained, and probed with PI LPS-specific mAbs (10B5 and 10G7). e Standard curve for the quantification of C. burnetii Xinqiao strain by Coxiella-UPT-LF, with the logarithm of the difference between the T/C ratio and the cut-off value on the horizontal axis and the logarithm of the concentration $(\mathrm{GE} / \mathrm{ml})$ on the vertical axis. Data were expressed as mean $\pm \mathrm{SD}, n=3$. f Standard curve for the quantification of NMI LPS by Coxiella-UPT-LF, with the logarithm of the difference between the $\mathrm{T} / \mathrm{C}$ ratio and the cut-off value on the horizontal axis and the logarithm of the concentration (GE/ml) on the vertical axis. Data were expressed as mean $\pm S D, n=3$

To assess the influence of culture methods on Coxiella-UPT-LF, six C. burnetii strains, i.e., Xinqiao, Qiyi, Yaan, H-11, NMII, and Grita, were cultured in YS, transferred to acidified citrate cysteine medium-2 (ACCM-2), cultured for another five passages, and then detected by Coxiella-UPT-LF. However, the C. burnetii Qiyi strain grew poorly in ACCM-2 medium, as has previously been reported for other strains as well [13, 14]. The sensitivity of Coxiella-UPT-LF for C. burnetii Xinqiao, Yaan, and $\mathrm{H}-11$ cultured in ACCM-2 was less than $1 \times 10^{6}, 1 \times 10^{6}$, and $1 \times 10^{7} \mathrm{GE} / \mathrm{ml}$, respectively (Fig. 4b), which is slightly lower than or equal to that of bacteria in YS suspension.

The inclusivity of mAbs was further confirmed by immunoblotting with LPS of C. burnetii PI strains that were extracted from YS culture of C. burnetii Xinqiao and cell-free culture of Xinqiao, Yaan, and H-11. As shown in Fig. 4c (also shown in Fig. S4 and Fig. S5), mAbs pooled from 10B5 and 10G7 reacted with LPS of these strains, as indicated by a laddering profile above $14 \mathrm{kDa}$. 


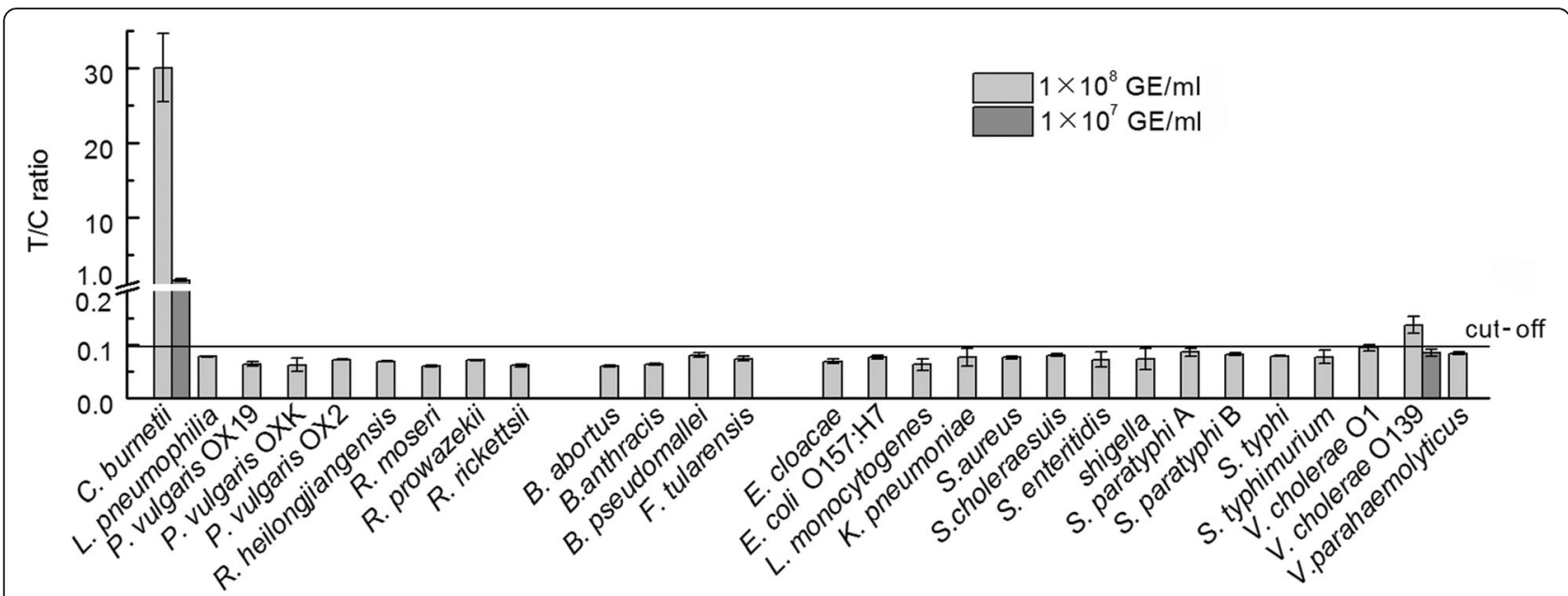

Fig. 3 Evaluation of the specificity of Coxiella-UPT-LF. Except slight cross-reaction with V. cholerae O139, Coxiella-UPT-LF showed high specificity, without false-positive results for non-specific bacterial species. Data were expressed as mean $\pm S D, n=3$

\section{Detection of $C$. burnetii from tissues of challenged mice and tick samples}

To assess whether the Coxiella-UPT-LF assay can be used for the detection of infected samples, mice were challenged with $10^{8}$ GEs of C. burnetii Xinqiao strain purified from YS. On day 7 post-challenge, mice were killed, and tissues were analysed by Coxiella-UPT-LF assay. Tissues from healthy mice served as negative controls (Fig. 5a). For healthy mice, organ suspensions with 100- and 1000-fold dilutions have little influence on the Coxiella-UPT-LF assay, but T/C ratios of 10 -fold dilutions of heart, liver, and lung suspensions were slightly higher than the cut-off value and were identified as false-positive results. For challenged mice, organ suspensions with 10-, 100-, and 1000-fold dilutions all tested positive, inferring that 1000-fold dilutions are suitable for $C$. burnetii detection. In addition, the numbers of $C$. burnetii gene copies in undiluted organ suspensions ranged from $1.7 \times 10^{8}$ to $2.6 \times 10^{9} \mathrm{GE} / \mathrm{ml}$ (Table S1), so the detection limits of the UPT-LF assay for $C$. burnetii in infected mouse samples may be more than $1 \times 10^{5} \mathrm{GE} / \mathrm{ml}$ (the detection limit obtained in vitro). However, false positive results were observed when blood samples of both challenged and healthy mice were tested (Table S1). This was due to the signal reduction in the control line caused by antibodies in mouse blood that were irrelevant to $C$. burnetii. These antibodies reacted with the goat anti-mouse secondary antibodies on control line of Coxiella-UPT-LF which were intended to be combined with UCP-mAbs.

Moreover, ticks with sucked blood were collected from the field for evaluation of the Coxiella-UPT-LF assay. Thirty-five samples, with each sample containing about 228 ticks, were homogenised in PBS and analysed. PBS has little impact on Coxiella-UPT-LF, as its detection results are similar to those of non-saline phosphate buffer. As shown in Fig. 5b, 8 of 35 samples tested positive. The numbers of $C$. burnetii gene copies in these eight samples ranged from $2.4 \times 10^{5}$ to $6.2 \times 10^{6} \mathrm{GE} / \mathrm{ml}$, as quantified by real-time PCR (Table S2), indicating that the detection limit of the UPT-LF assay for $C$. burnetii in naturally infected tick samples is higher than $2 \times 10^{5} \mathrm{GE} / \mathrm{ml}$.

\section{Discussion}

Several mAbs against $C$. burnetii have previously been developed for diagnostic or vaccine use. A PI LPS targeting $\mathrm{mAb}$ and its variable fragments were developed for prophylaxis against $C$. burnetii exposure by Peng et al. [15]. A PI LPS targeting mAb was also developed by Palkovicova et al. for the detection of the virulent form of C. burnetii and for differentiation of the individual isolates $[16,17]$. However, data on mAb-based assays for the quantitative detection of $C$. burnetii are rare. Therefore, in this study, we aimed to establish an mAb-based UPT-LF assay for the rapid and quantitative detection of C. burnetii PI strains, especially for use in the field.

In our Coxiella-UPT-LF assay, the detection limits were $10^{4} \mathrm{GE} / \mathrm{ml}$ for purified C. burnetii PI and $10 \mathrm{ng} / \mathrm{ml}$ for purified NMI LPS. Using $0.1 \mathrm{ml}$ loading volume, the actual final amounts applied to the strip required for detection are 1000 C. burnetii bacteria and $1 \mathrm{ng}$ of NMI LPS, indicating very high sensitivity for an immune-chromatography method used for rapid screening. The detection limits of CoxiellaUPT-LF for C. burnetii in YS suspension, in experimentally infected mice, and in naturally infected ticks were more than $10^{5} \mathrm{GE} / \mathrm{ml}$, i.e., at least 10 times higher than that for the detection of purified $C$. burnetii. These results indicated that Coxiella-UPT-LF cannot fully distinguish C. burnetii from culture medium components or animal tissues. 

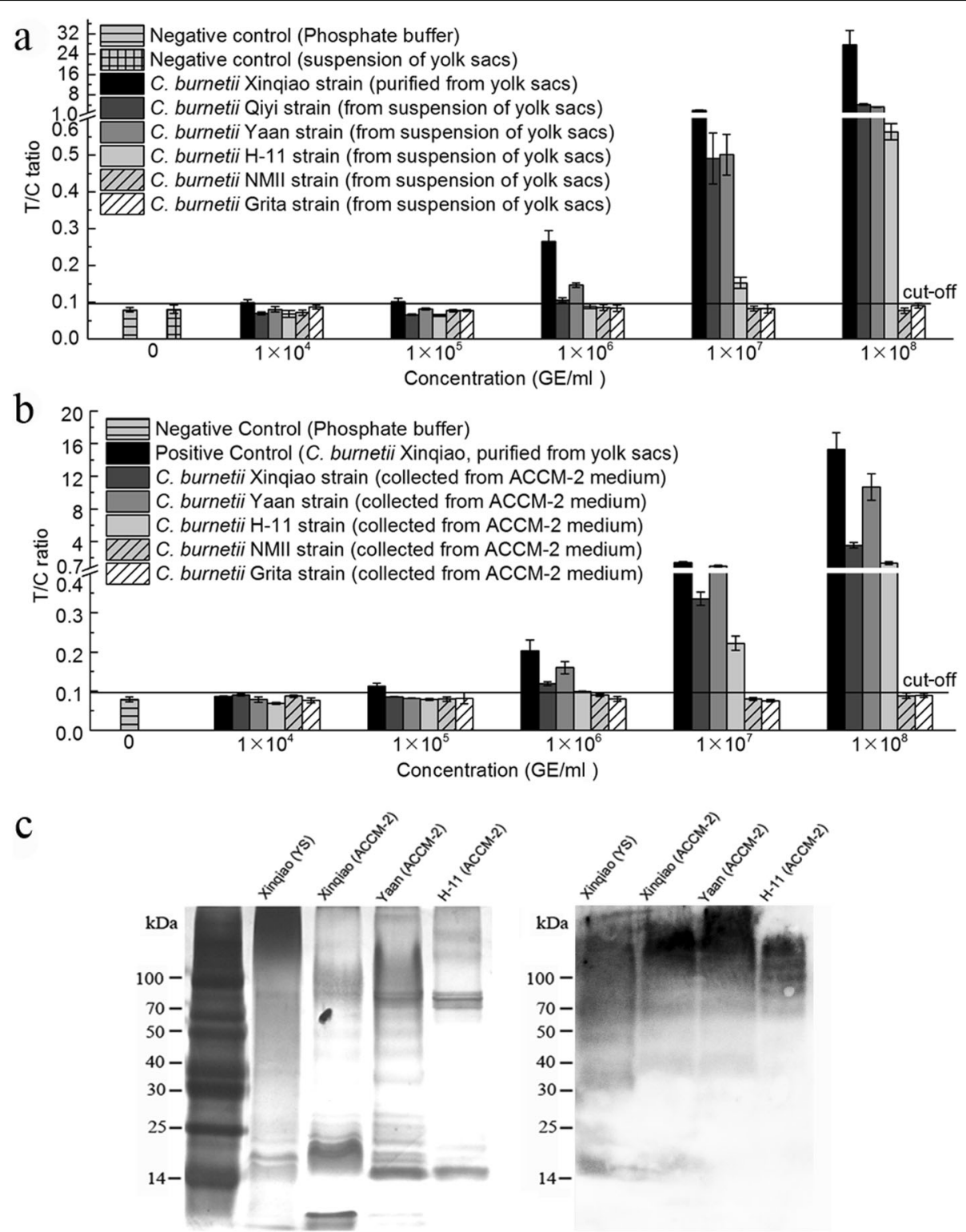

Fig. 4 Inclusivity of Coxiella-UPT-LF for C. burnetii. a The inclusivity of Coxiella-UPT-LF for the detection of C. burnetii strains cultured in YS. Data were expressed as mean $\pm S D, n=3$. b The inclusivity of Coxiella-UPT-LF for the detection of C. burnetii strains cultured in ACCM-2 medium. Data were expressed as mean $\pm S D, n=3$. c LPS immunoblot of $C$. burnetii PI strains isolated in China. LPS of C. burnetii Xinqiao, Yaan, and H-11 strains was separated by SDS-PAGE, silver-stained, and probed with PI LPS-specific mAbs (10B5 and 10G7)

C. burnetii Xinqiao, Yaan, Qiyi, and H-11 strains were isolated from China and characterised as PI strains [18]. Coxiella-UPT-LF recognised all these strains with different sensitivities, and additionally recognised LPS extracted from C. burnetii NMI, indicating that different isolates of C. burnetii PI strains may share the same LPS or other antigens, even though they differed in size and composition [3]. In addition, the Coxiella-UPT-LF assay showed good inclusivity for the detection of various isolates of C. burnetii PI strains, which might be of great importance when the assay is applied in the field, where no information on the isolates is available.
The sensitivity of the assay for the detection of Xinqiao strain purified from ACCM-2 medium was lower than that for the detection of the same strain purified from YS. In addition, PII-specific bands were observed in the LPS profiles of cell-free cultured Xinqiao strains. These results indicate that $C$. burnetii PI strains were undergoing phase transition when serially passaged in artificial medium [3, 14].

The physiology, lifestyle, and morphology of Rickettsia species are similar to those of C. burnetii, and Legionella pneumonia is closely related to C. burnetii, sharing genomic homology and causing similar clinical presentations. 


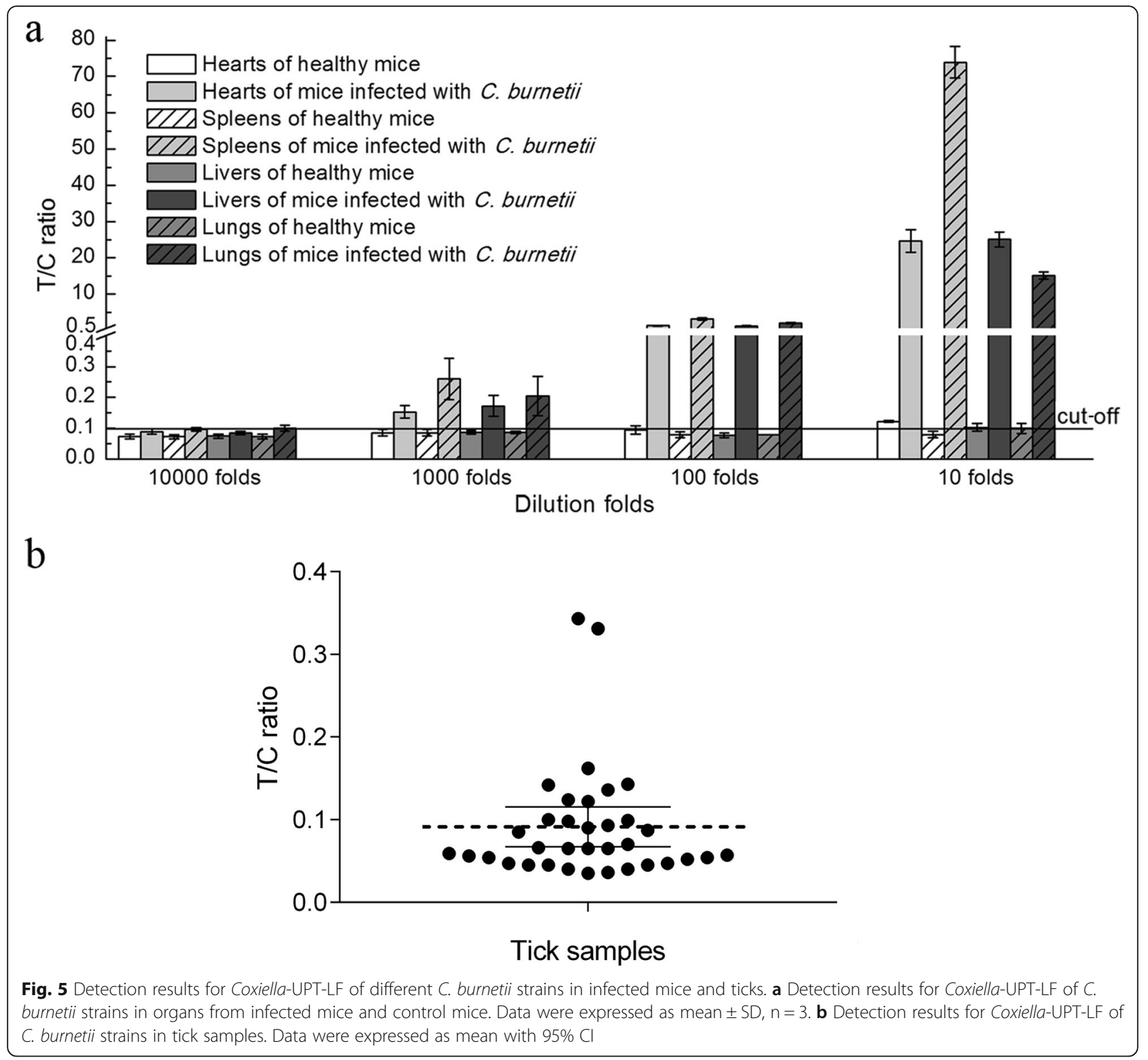

In addition to the above-mentioned bacteria, the conditioned pathogen Proteus vulgaris, which shares genomic homology with C. burnetii, bioterrorism agents, and foodborne bacteria that have similar habitats as $C$. burnetii must be distinguished from C. burnetii in the CoxiellaUPT-LF assay. The Coxiella-UPT-LF assay gave negative results for these bacterial strains, even at $10^{8} \mathrm{GE} / \mathrm{ml}$, confirming the high specificity of the assay.

\section{Conclusions}

Overall, the Coxiella-UPT-LF assay exhibited good sensitivity and specificity for the detection of $C$. burnetii PI strains in culture medium and in experimentally and naturally infected samples. Although further optimisation is necessary, Coxiella-UPT-LF is a reliable method for the rapid screening of $C$. burnetii and is suitable for on-site detection in the field.

\section{Methods}

Ethics

Thirteen female BALB/c mice were used for the preparation of $\mathrm{mAb}$ and infection with C. burnetii. This study complied with the Guidelines for the Welfare and Ethics of Laboratory Animals of China and was approved by the Committee of Welfare and Ethics of Laboratory Animals, Beijing Institute of Microbiology and Epidemiology (permit number: AMMS-2014-025). 


\section{Bacteria, samples, and materials}

All bacteria were preserved in our laboratory. C. burnetii PI strains that isolated in China, including Xinqiao, Qiyi, Yaan, and H-11 strain [18, 19], as well as C. burnetii PII strains, including NMII and Grita strain, were initially propagated in YS for 7-9 passages. Then the strains were transferred to ACCM-2 for another five passages in order to adapt to the cell-free culture system and to synchronise the passage numbers of the strains [20,21]. Purified $C$. burnetii Xinqiao strain was separated from YS by renografin density centrifugation as previously described [22]. The concentrations of $C$. burnetii were determined by realtime PCR detecting the com 1 gene as previously described [23]. Purified LPS of NMI and NMII was provided by Dr. Chen Chen from the University of California, Berkeley as a generous gift. The LPS of other $C$. burnetii strains was extracted using a modified hot phenol method and analysed by SDS-PAGE as previously described [3].

Legionella pneumophila was cultured on BCYE agar plates. Bacterial suspensions of $P$. vulgaris OXK, $P$. vulgaris OX19, and $P$. vulgaris OX2 were purchased from Ningbo Tianrun Bio-Pharmaceutical Co. Ltd. (Ningbo, China). Rickettsia moseri, Rickettsia prowazekii, Rickettsia rickettsii, and Rickettsia heilongjiangensis were cultured in Vero cells or embryo cells and purified by differential centrifugation followed by centrifugation through 30\% renografin [24]. Bioterrorism agents and the food-borne bacteria were all cultured in lysogeny broth, except for Francisella tularensis, Brucella abortus, and Burkholderia pseudomallei, which were cultured in brain heart infusion media. The concentrations of the bacteria were determined by real-time PCR.

UCP particles were purchased from Shanghai Kerune Phosphor Technology Co., Ltd. (Shanghai, China) and modified by our laboratory for combination with antibodies. Glass fibre and nitrocellulose membrane were purchased from Millipore Corp. (Bedford, MA, USA). Absorbent pad was purchased from Shanghai Goldbio Technology Co. Ltd. (Shanghai, China). The UPT biosensor was obtained from the Shanghai Institute of Optics and Fine Mechanics, the Chinese Academy of Sciences (Shanghai, China).

\section{mAb generation and sequence analysis}

To generate mAbs against $C$. burnetii, five BALB/c mice were immunised with $20 \mu \mathrm{g}$ of formalin-inactivated $C$. burnetii Xinqiao PI antigen four times at 3-week intervals. Then they were humanely sacrificed by cervical dislocation, and splenocytes were isolated. The positive hybridomas were obtained from the fusion of splenocytes from PI-immunised mice with SP2/0 myeloma cells and screened by ELISA for their ability to react with PI and PII antigens, as described previously [15]. Ascites were obtained by intraperitoneal injection of hybridomas into BALB/c mice, and antibodies in ascites were purified by caprylic acid/saturated ammonium sulphate precipitation. The antibody concentrations were determined by ultraviolet spectrophotometry, and their potencies were measured through serial two-fold dilutions. Finally, five antibodies were primarily selected: 10B5, 10G7, 13D6, 6D8, and 8A1.

To determine the cDNA-derived amino acid sequences of the $\mathrm{VH}$ and VL chains of these mAbs, total RNA was extracted from $10^{6} \mathrm{mAb}$ producing hybridoma cells using the Qiagen RNeasy Mini Kit (Qiagen, Valencia, CA). The cDNA of hybridoma cells was synthesised by the $5^{\prime}$-RACE method. The genes of $\mathrm{VH}$ and VL were amplified from the synthesised CDNA by PCR and sequenced by Genewiz Co. Ltd. (Suzhou, China). The sequences were compared with the mouse IgG database using IMGT/V-Quest (www.imgt.cines.fr/home.html). The automatic modelling of $\mathrm{mAb}$ variable domains was established by the canonical structure method from abYmod (http://abymod.abysis.org/).

\section{Establishment of the UPT assay}

The $\mathrm{mAbs}(2 \mathrm{mg} / \mathrm{ml})$ against $C$. burnetii and goat antimouse were dispensed at a speed of $1 \mu \mathrm{l} / \mathrm{cm}$ as test line $(\mathrm{T})$ and control line $(\mathrm{C})$ on nitrocellulose membranes. UCPs were conjugated with different antibodies to prepare different conjugation pads. The nitrocellulose membrane with each antibody was combined with various conjugation pads, attached to a sticky base with absorbent papers and sample pads, and finally cut into $4 \mathrm{~mm}$ pieces to fabricate different UPT strips.

The primary sample treating buffer $(0.03 \mathrm{M}$ phosphate buffer containing $0.5 \%$ IGEPAL CA-630) was used for the selection of antibodies suitable for UPT detection and mixed with C. burnetii Xinqiao strain purified from YS at serial concentrations $\left(10^{7}, 10^{8}\right.$, and $\left.10^{9} \mathrm{GE} / \mathrm{ml}\right)$ at a ratio of 9:1 before loading to each strip. As a negative control, $0.03 \mathrm{M}$ phosphate buffer was used. After $15 \mathrm{~min}$, the strip was put into the UPT biosensor to read the signal intensities of the $\mathrm{T}$ and $\mathrm{C}$ bands. The $\mathrm{T} / \mathrm{C}$ ratios were used as the detection results. The results of $C$. burnetii and phosphate buffer samples were abbreviated as $\mathrm{P}$ and $\mathrm{N}$. To evaluate the performance of each UPT-LF strip (fabricated with different antibodies), the $(\mathrm{P}-\mathrm{N}) / \mathrm{N}$ ratio was calculated. The UPT-LF strips with the highest $(\mathrm{P}-\mathrm{N}) / \mathrm{N}$ ratios were selected for subsequent experiments.

The method for labelling UCPs and antibodies and the components of sample treating buffer were further optimised. The final optimised sample treating buffer was dispensed on the conjugation pad and dried at $37^{\circ} \mathrm{C}$ for $1 \mathrm{~h}$. Samples could be directly applied on the strips.

\section{Evaluation of detection limit, sensitivity, and precision}

To determine the cut-off value of Coxiella-UPT-LF, $0.1 \mathrm{ml}$ of $0.03 \mathrm{M}$ phosphate buffer was applied to the strip. 
Phosphate buffer was also used as a negative control. $C$. burnetii Xinqiao strain purified from YS was diluted in $0.03 \mathrm{M}$ phosphate buffer into $1 \times 10^{3}, 5 \times 10^{3}, 1 \times 10^{4}, 5 \times$ $10^{4}, 1 \times 10^{5}, 5 \times 10^{5}, 5 \times 10^{6}, 5 \times 10^{7}$, and $1 \times 10^{8} \mathrm{GE} / \mathrm{ml}$, while LPS from NMI and NMII was diluted to 1, 10, 100, 1000, and $10,000 \mathrm{ng} / \mathrm{ml}$ for UPT detection. Each dilution was added to three Coxiella-UPT-LF strips.

For LPS immunoblotting, C. burnetii LPS was extracted, transferred to a PVDF membrane, and incubated with pooled 10G7 and 10B5 mAbs at 1:10,000 dilution and then HRP-conjugated goat anti-mouse IgM. LPS was detected by chemiluminescence as previously described [3].

\section{Evaluation of specificity}

Numerous bacteria at a concentration of $10^{8} \mathrm{GE} / \mathrm{ml}$ were used to evaluate the specificity of Coxiella-UPT-LF. Genetically related bacteria included L. pneumophila, $P$. vulgaris OXK, $P$. vulgaris OX19, $P$. vulgaris OX2, $R$. heilongiangensis, $R$. moseri, $R$. prowazekii, and $R$. rickettsii. Bioterrorism agents included B. abortus, Bacillus anthracis, B. pseudomallei, and F. tularensis. The food-borne pathogens with similar habitats to C. burnetii included Enterobacter cloacae, Escherichia coli O157, Listeria monocytogenes, Klebsiella pneumoniae, Staphylococcus aureus, Salmonella enterica serotype choleraesuis, Salmonella enteritidis, Shigella, Salmonella paratyphi A, S. paratyphi B, Salmonella typhi, Salmonella typhimurium, $V$. cholerae $\mathrm{O} 1, V$. cholerae $\mathrm{O} 139$, and Vibrio parahaemolyticus. The bacterial samples were diluted in $0.03 \mathrm{M}$ phosphate buffer and directly applied to the Coxiella-UPT-LF assay. Each sample was tested in triplicate.

\section{Evaluation of inclusivity}

To assess the capacity of Coxiella-UPT-LF to detect diverse C. burnetii strains, three PI strains of $C$. burnetii were employed, i.e., C. burnetii Qiyi, Yaan, and H-11, in YS suspension. To evaluate the influences of culture methods on the detection, $C$. burnetii strains cultured in ACCM-2 medium were collected through centrifugation. All of the bacterial samples were diluted to final concentrations of $1 \times 10^{4}, 1 \times 10^{5}, 1 \times 10^{6}, 1 \times 10^{7}$, and $1 \times 10^{8}$ $\mathrm{GE} / \mathrm{ml}$ in $0.03 \mathrm{M}$ phosphate buffer. The concentrations were confirmed by real-time PCR detection of the com 1 gene of C. burnetii [23]. Then, $0.1 \mathrm{ml}$ of the prepared samples was applied to Coxiella-UPT-LF strips. Suspension of normal yolk sac was also diluted 10-, 100-, 1000-, and 10, 000-fold to evaluate its influence on the detection of Coxiella-UPT-LF. Each sample was tested in triplicate.

\section{Application of the Coxiella-UPT-LF assay to experimentally and naturally infected samples} Tissues of mice challenged with $C$. burnetii were used as infected samples for the evaluation of Coxiella-UPT-LF.
Eight female, 6-week-old BALB/c mice were randomly divided into two groups. In the infected group, five mice were infected with $10^{8} \mathrm{GE}$ C. burnetii Xinqiao strain through intraperitoneal injection. In the control group, three mice were injected with PBS. One week after infection, mice were humanely sacrificed by cervical dislocation, and their spleens, lungs, livers, hearts and blood were collected. Then the organs were homogenised into cell suspensions and the whole blood was lysed into suspensions. The suspensions were diluted 10-, 100-, and 1000 -fold in $0.03 \mathrm{M}$ phosphate buffer for sample preparation, and then $0.1 \mathrm{ml}$ was directly applied to the strip. The DNA from organ suspensions was extracted using the DNeasy Blood \& Tissue Kit (QIAGEN), and the bacterial burden was quantified by real-time PCR as described above.

Eight thousand ticks with sucked blood were collected from the forest steppe field in public places of Tianshan Mountain, Wusu City, Xinjiang province, China. They were divided into 35 portions. Each portion, with about 228 ticks, was ground into powder and vortexed into suspension in PBS. The suspensions were deposited for 2-4 min, and the supernatants were directly applied in Coxiella-UPT-LF, with PBS as a negative control. The DNA of each sample was extracted using the DNeasy Blood \& Tissue Kit (QIAGEN), and the bacterial burden was quantified by real-time PCR as described above.

\section{Supplementary information}

Supplementary information accompanies this paper at https://doi.org/10. 1186/s12866-020-01934-0.

\section{Additional file 1: Figure S1. Amino acid sequence and models of variable domains of $10 B 5$ and 10G7. (a) Analysis of amino acid sequence of $\mathrm{VL}$ chains of 10B5 and 10G7; (b) Analysis of amino acid sequence of $\mathrm{VH}$ chains of $10 \mathrm{~B} 5$ and $10 \mathrm{G} 7$; (c) Models of variable domains of $10 \mathrm{~B} 5$ and 10G7 established by the molecular modeling.}

Additional file 2: Figure S2. Raw image of LPS profile of C. burnetii $\mathrm{NMI}$ and NMII determined by silver stain.

Additional file 3: Figure S3. Raw image of LPS profile of C. burnetii $\mathrm{NMI}$ and NMII determined by immunoblot.

Additional file 4: Figure S4. Raw image of LPS profile of C. burnetii PI strains isolated in China determined by silver stain.

Additional file 5: Figure S5. Raw image of LPS profile of C. burnetii PI strains isolated in China determined by immunoblot.

Additional file 6: Table S1. The T/C ratios of UPT-LF and the C. burneti gene copies for organ suspensions of infected mice.

Additional file 7: Table S2. The T/C ratios of UPT-LF and the C. burnetii gene copies for tick samples.

\section{Abbreviations}

ACCM-2: Acidified citrate cysteine medium-2; C. burnetii: Coxiella burnetii; IFA: Immunofluorescence assay; LPS: Lipopolysaccharide; NMI: Nine Mile phase I strain; NMII: Nine Mile phase II strain; PI: Phase I; PII: Phase II; POCT: Point-of-care testing; UCPs: Up-converting phosphor particles; UPTLF: Up-converting phosphor technology-based lateral flow; YS: Yolk sacs; VH: Variable heavy; VL: Variable light 


\section{Acknowledgments}

We thank Dr. Chen Chen from the University of California, Berkeley for providing purified LPS of NMI and NMII. We thank LetPub (www.letpub.com) for its linguistic assistance during the preparation of this manuscript.

\section{Authors' contributions}

PPZ, JJ, YZ, RFY, XLX: data acquisition, data analysis, data interpretation, writing of the manuscript, revising of the manuscript; MJF, JW, YQW: reagent provision, data analysis, revising of the manuscript; YJS, DSZ, BHW: revising of the manuscript. The authors have read and approved the manuscript.

\section{Funding}

This work was supported by the Beijing Nova Program (Z181100006218116), the National Key Research and Development Program of China (2018YFC1200502), the National Natural Science Foundation of China (31970178) and the Beijing Natural Science Foundation (5204039). The funders had no role in study design, data collection and analysis, decision to publish, or preparation of the manuscript.

\section{Availability of data and materials}

All data generated or analyzed during current study are available from the corresponding author on reasonable request.

\section{Ethics approval and consent to participate}

This study complied with the Guidelines for the Welfare and Ethics of Laboratory Animals of China, and was approved by The Committee of Welfare and Ethics of Laboratory Animal, Beijing Institute of Microbiology and Epidemiology (Permit number: AMMS-2014-025).

\section{Consent for publication}

Not applicable.

\section{Competing interests}

Author Dongsheng Zhou is an Associate Editor of this journal. The authors have no conflict of interest to declare.

\section{Author details}

'State Key Laboratory of Pathogen and Biosecurity, Beijing Institute of Microbiology and Epidemiology, Beijing, P. R. China. ${ }^{2}$ Beijing Key Laboratory of POCT for Bio-emergency and Clinic (No.BZ0329), Beijing, P. R. China. ${ }^{3}$ Preventive Veterinary Medicine, College of Veterinary Medicine, China Agricultural University, Beijing, P. R. China.

Received: 7 February 2020 Accepted: 3 August 2020

Published online: 12 August 2020

\section{References}

1. Amano K, Williams JC. Chemical and immunological characterization of lipopolysaccharides from phase I and phase II Coxiella burnetii. J Bacteriol. 1984;160(3):994-1002.

2. Hackstadt T, Peacock MG, Hitchcock PJ, Cole RL. Lipopolysaccharide variation in Coxiella burnetti: intrastrain heterogeneity in structure and antigenicity. Infect Immun. 1985;48(2):359-65.

3. Beare PA, Jeffrey BM, Long CM, Martens CM, Heinzen RA. Genetic mechanisms of Coxiella burnetii lipopolysaccharide phase variation. PLoS Pathog. 2018;14(3):e1006922.

4. Raoult D, Marrie T, Mege J. Natural history and pathophysiology of Q fever. Lancet Infect Dis. 2005;5(4):219-26.

5. Bae M, Jin CE, Park JH, Kim MJ, Chong YP, Lee SO, et al. Diagnostic usefulness of molecular detection of Coxiella burnetii from blood of patients with suspected acute Q fever. Medicine. 2019;98(23):e15724.

6. Schneeberger PM, Hermans MH, van Hannen EJ, Schellekens JJ, Leenders $A C$, Wever PC. Real-time PCR with serum samples is indispensable for early diagnosis of acute $\mathrm{Q}$ fever. Clin Vaccine immunol. 2010;17(2):286-90.

7. Hendrix $L R$, Chen $C$. Antigenic analysis for vaccines and diagnostics. Adv Exp Med Biol. 2012:984:299-328.

8. Fournier PE, Raoult D. Comparison of PCR and serology assays for early diagnosis of acute Q fever. J Clin Microbiol. 2003:41(11):5094-8.

9. Zhao $Y$, Wang $H$, Zhang $P$, Sun $C$, Wang $X$, Wang $X$, et al. Rapid multiplex detection of 10 foodborne pathogens with an up-converting phosphor technology-based 10-channel lateral flow assay. Sci Rep. 2016;6:21342.
10. Hao M, Zhang P, Li B, Liu X, Zhao Y, Tan H, et al. Development and evaluation of an up-converting phosphor technology-based lateral flow assay for the rapid, simultaneous detection of Vibrio cholerae serogroups $\mathrm{O1}$ and O139. Sci Rep. 2017;12(6):e0179937.

11. Hua F, Zhang P, Zhang F, Zhao Y, Li C, Sun C, et al. Development and evaluation of an up-converting phosphor technology-based lateral flow assay for rapid detection of Francisella tularensis. Sci Rep. 2015;5:17178.

12. Zhang P, Liu X, Wang C, Zhao Y, Hua F, Li C, et al. Evaluation of upconverting phosphor technology-based lateral flow strips for rapid detection of Bacillus anthracis Spore, Brucella spp., and Yersinia pestis. PLoS One. 2014;9(8):e105305.

13. Kersh GJ, Priestley RA, Hornstra HM, Self JS, Fitzpatrick KA, Biggerstaff BJ, et al. Genotyping and axenic growth of Coxiella burnetii isolates found in the United States environment. Vector Borne Zoonotic Dis (Larchmont, NY). 2016:16(9):588-94.

14. Kersh GJ, Oliver LD, Self JS, Fitzpatrick KA, Massung RF. Virulence of pathogenic Coxiella burnetii strains after growth in the absence of host cells. Vector Borne Zoonotic Dis (Larchmont, NY). 2011;11(11):1433-8.

15. Peng Y, Schoenlaub L, Elliott A, Mitchell WJ, Zhang G. Characterization of a lipopolysaccharide-targeted monoclonal antibody and its variable fragments as candidates for prophylaxis against the obligate intracellular bacterial pathogen Coxiella burnetii. Infect Immun. 2014:82(11):4530-41.

16. Palkovicova $K$, Ihnatko R, Vadovic P, Betinova E, Skultety L, Frangoulidis $D$, et al. A monoclonal antibody specific for a unique biomarker, virenose, in a lipopolysaccharide of Coxiella burnetii. Clin Microbiol Infect. 2009;15(Suppl 2): 183-4.

17. Narasaki CT, Toman R. Lipopolysaccharide of Coxiella burnetii. Adv Exp Med Biol. 2012;984:65-90.

18. Wen BH, Yu SR, Yu GQ, Li QJ, Zhang X. Analysis of proteins and lipopolysaccharides from Chinese isolates of Coxiella burnetii with monoclonal antibodies. Acta Virol. 1991;35(6):538-44.

19. Feng J, Hu X, Fu M, Dai L, Yu Y, Luo W, et al. Enhanced protection against $Q$ fever in BALB/c mice elicited by immunization of chloroform-methanol residue of Coxiella burnetii via intratracheal inoculation. Vaccine. 2019;37(41): 6076-84.

20. Omsland A, Beare PA, Hill J, Cockrell DC, Howe D, Hansen B, et al. Isolation from animal tissue and genetic transformation of Coxiella burnetii are facilitated by an improved axenic growth medium. Appl Environ Microbiol. 2011;77(11):3720-5.

21. Kuley R, Smith HE, Frangoulidis D, Smits MA, Jan Roest HI, Bossers A. Cellfree propagation of Coxiella burnetii does not affect its relative virulence. PLoS One. 2015;10(3):e0121661.

22. Williams JC, Peacock MG, McCaul TF. Immunological and biological characterization of Coxiella burnetii, phases I and II, separated from host components. Infect Immun. 1981;32(2):840-51

23. Lockhart MG, Graves SR, Banazis MJ, Fenwick SG, Stenos J. A comparison of methods for extracting DNA from Coxiella burnetii as measured by a duplex qPCR assay. Lett Appl Microbiol. 2011:52(5):514-20.

24. Clark TR, Lackey AM, Kleba B, Driskell LO, Lutter El, Martens C, et al. Transformation frequency of a mariner-based transposon in Rickettsia rickettsii. J Bacteriol. 2011:193(18):4993-5.

\section{Publisher's Note}

Springer Nature remains neutral with regard to jurisdictional claims in published maps and institutional affiliations.

Ready to submit your research? Choose BMC and benefit from:

- fast, convenient online submission

- thorough peer review by experienced researchers in your field

- rapid publication on acceptance

- support for research data, including large and complex data types

- gold Open Access which fosters wider collaboration and increased citations

- maximum visibility for your research: over $100 \mathrm{M}$ website views per year

At $\mathrm{BMC}$, research is always in progress.

Learn more biomedcentral.com/submission 